\title{
A influência da memória operacional nas habilidades de compreensão de leitura em escolares de $4^{\mathrm{a}}$ série
}

\author{
Influence of working memory in reading comprehension in 4th grade \\ students
}

\author{
Micheli Cristina Paludeto Belaz Giangiacomo ${ }^{1}$, Ana Luiza Gomes Pinto Navas ${ }^{2}$
}

\begin{abstract}
RESUMO
Objetivos: Investigar o papel da memória verbal e não-verbal, e do vocabulário expressivo na compreensão de leitura; avaliar as habilidades de memória e vocabulário em um grupo de crianças da $4^{\mathrm{a}}$ série em relação ao desempenho de compreensão de leitura. Métodos: Participaram do estudo 15 crianças com idades entre 9;11 anos e 11;4 anos. As crianças não possuíam queixas de perda auditiva, visual e/ou alterações neurológicas. Todos os sujeitos foram submetidos individualmente às avaliações de desempenho escolar, leitura, (decodificação, compreensão) vocabulário e memória operacional verbal e não-verbal. Resultados: Houve correlação estatisticamente significante entre o conhecimento de vocabulário expressivo $(r=0,546, p=0,035)$ e memória operacional verbal $(\mathrm{r}=-0,689, \mathrm{p}=0,004)$ e o desempenho em compreensão neste grupo de crianças de $4^{\mathrm{a}}$. série de ensino fundamental, sem queixas de dificuldades escolares. Nem a memória não-verbal, nem a habilidade de decodificação de palavras influenciaram no desempenho em compreensão de leitura. Conclusões: O desempenho em vocabulário e a boa capacidade da memória operacional verbal foram fatores relevantes para garantir a compreensão de leitura nessa população.
\end{abstract}

Descritores: Leitura; Memória; Compreensão; Estudantes; Educação primária e fundamental; Criança

\section{INTRODUÇÃO}

A leitura implica um componente de decodificação e de compreensão. Quando se aprende a ler, o leitor principiante pode decodificar palavras em um texto, mas não significa que esteja ocorrendo compreensão do que está sendo $\operatorname{lido}^{(1)}$. Em muitos casos, mesmo quando a leitura de palavras é eficiente não há compreensão, pois tanto a decodificação como a compreensão podem estar comprometidas.

Para a compreensão da leitura de textos é exigido do leitor que ele tenha habilidades de reconhecimento de palavras e a integração de seu significado ${ }^{(2)}$. É necessário que o leitor iniciante desenvolva, entre outras habilidades, um amplo vocabulário e aprenda a ler com fluência.

Trabalho realizado no Curso de Especialização em Linguagem da Faculdade de Ciências Médicas da Santa Casa de São Paulo - FCMSCSP - São Paulo (SP), Brasil.

(1) Especialista em Linguagem pela Faculdade de Ciências Médicas da Santa Casa de São Paulo - FCMSCSP - São Paulo (SP), Brasil; Fonoaudióloga clínica - Tietê (SP), Brasil.

(2) Professora adjunto do Curso de Fonoaudiologia da Faculdade de Ciências Médicas da Santa Casa de São Paulo - FCMSCSP - São Paulo (SP), Brasil.

Endereço para correspondência: Ana Luiza Gomes Pinto Navas. R. Dr. Cesário Motta Jr, 61, Santa Cecília, São Paulo - SP, CEP 01221-020. E-mail: analunavas@gmail.com

Recebido em: 3/12/2007; Aceito em: 29/2/2008
Alguns fatores contribuem para a compreensão da leitura: a eficiência de decodificação ${ }^{(3)}$, domínio do conhecimento, vocabulário $^{(3)}$, capacidade de fazer inferências ${ }^{(4-5)} \mathrm{e}$ fatores sociais. Assim, a compreensão é um processo ativo que requer uma interação intencional e cuidadosa entre o leitor e o texto ${ }^{(6)}$.

A memória também possui um papel importante; por meio da memória evoca-se todo o conhecimento adquirido e armazenado (memória de longo prazo) para que possa ser usado na compreensão da leitura. Além disso, é na memória operacional que as palavras lidas são armazenadas até haver o processamento do significado. A memória operacional participa ativamente na aquisição de vários conhecimentos ${ }^{(7)}$. É por meio dessa memória que ocorre o armazenamento e a manipulação de informações durante um período curto de tempo, sendo necessária para o funcionamento cognitivo e efetivo das atividades coloquiais como o rendimento escolar e profissional ${ }^{(8)}$.

A relação entre compreensão de leitura e memória operacional é demonstrada em vários estudos ${ }^{(9-14)}$. No entanto, a natureza e modos de avaliação da memória são bastante diversos e deve-se ter clareza da natureza da memória e dos estímulos empregados nesta avaliação.

Desse modo, é importante identificar até que ponto a função da memória operacional se relaciona com a habilidade de compreensão de textos e com o desempenho em habilidades escolares, para que sejam desenvolvidos programas de 
prevenção e intervenção que priorizem estes aspectos para a realidade brasileira.

Este trabalho teve por objetivo investigar o papel da memória verbal e não-verbal na compreensão de leitura. Os objetivos específicos foram: (a) avaliar as habilidades de memória verbal e não-verbal em um grupo de crianças de $4^{\mathrm{a}}$ série; (b) estabelecer a relação entre a memória operacional verbal e não verbal, vocabulário e a compreensão de leitura.

\section{MÉTODOS}

Esta pesquisa foi aprovada pelo Comitê de Ética em Pesquisa da Irmandade da Santa Casa de Misericórdia de São Paulo sob o número do protocolo 477/06.

Participaram da pesquisa 15 crianças, sendo seis do sexo masculino e nove do sexo feminino, com idades entre 9;11 anos e 11;4 anos, regularmente matriculadas na $4^{\mathrm{a}}$ série do ensino fundamental de uma escola particular do interior do estado de São Paulo.

Não participaram da pesquisa crianças que possuíam queixas de perda auditiva e/ou alteração neurológica.

\section{Material}

Todas as crianças foram submetidas individualmente às avaliações de desempenho escolar, de leitura, de decodificação, compreensão e vocabulário bem como de memória operacional. Os professores responderam a um questionário que permitiu a classificação de seus alunos nas seguintes categorias de competência de leitura: a) lêem bem; b) lêem razoavelmente e c) lêem mal ${ }^{(15)}$.

A avaliação do desempenho escolar foi feita por meio do Teste de Desempenho Escolar (TDE) ${ }^{(16)}$, que avalia a escrita do nome e de palavras isoladas, leitura de palavras isoladas e aritmética, sendo que este último aspecto não foi realizado. A tarefa de escrita é feita a partir de um ditado de 34 palavras seguidas de frases, que as contextualizam, e para a escrita do nome, é marcado um ponto para cada item correto. Na tarefa de leitura, são apresentadas 70 palavras isoladas, e marcado um ponto para cada palavra lida corretamente.

O desempenho em leitura e escrita foi avaliado por tarefas que englobavam leitura de letras, palavras e pseudopalavras; ditado de letras, palavras e pseudopalavras; escrita de palavras e frases a partir de figura.

A avaliação do vocabulário foi feita com o ABFW - Teste de Linguagem infantil ${ }^{(17)}$. São abrangidos os seguintes campos conceituais: vestuário, animais, alimentos, meios de transporte, móveis e utensílios, profissões, locais, formas e cores, brinquedos e instrumentos musicais.

A avaliação da Compreensão de Leitura foi feita a partir de: (a) leitura e respostas às questões de dois textos - "O peixão e o peixinho" e "O vento e o sol", sendo que para cada texto havia 11 questões em que as crianças deveriam responder, sim ou não. A leitura foi gravada em fita cassete a fim de se estimar os parâmetros de duração da mesma. Foi medido o tempo de realização da tarefa e calculada a quantidade de palavras lidas por minuto (ppm); e (b) apresentação de texto cloze, "O amigo de Juliana", para completar as lacunas com as palavras adequadas, sendo que havia 13 lacunas e as respostas foram classificadas como acerto, substituição e erro. Também foi calculado o tempo de realização da tarefa em minutos.

Para a avaliação da memória operacional foram aplicados testes que avaliam a capacidade de memória verbal e nãoverbal. Foram utilizados os testes de (a) Memória seqüencial auditiva de palavras e pseudopalavras ${ }^{(18)}$; (b) Memória seqüencial não-verbal com apoio visual e (c) Teste de Repetição de Pseudopalavras $^{(19)}$.

O teste de memória seqüencial auditiva de palavras e pseudopalavras contém 60 estímulos cada um. Todos os estímulos são dissílabos paroxítonos com estrutura CVCV. Foram apresentadas quatro listas com três blocos de estímulos com duas, três, quatro, cinco e seis seqüências de palavras (L3 e L4) e pseudopalavras (L1 e L2) em cada bloco. O intervalo entre os estímulos foi de um segundo para as listas L1 e L4 e de 500 milisegundos para as listas L2 e L3. A apresentação foi feita por meio de $C D$ player com fones de ouvido e as respostas foram gravadas em fita cassete. A tarefa solicitada foi a de ouvir a sequiência de estímulos de um bloco e ao final repeti-los na ordem correta.

A avaliação de memória seqüencial não-verbal com apoio visual foi realizada com um jogo que produz diferentes sons em sequiência ao mesmo tempo em que as luzes dos botões correspondentes se acendem. A seqüência inicia-se com um estímulo e a cada acerto, um novo estímulo é acrescentado à sequiência anterior. Se houvesse erro o jogo se encerraria. Para a avaliação da memória não-verbal foi estabelecido o seguinte critério: foram três tentativas e o nível de memória não-verbal foi determinado pela média de acertos dessas.

O Teste de Repetição de Pseudopalavras é composto por 40 pseudopalavras divididas em grupo de dez conforme o número de sílabas (duas a cinco sílabas). As pseudopalavras foram lidas pela avaliadora sem o apoio da pista visual a fim de evitar leitura labial pelas crianças, e repetidas pelo sujeito uma a uma. A cada apresentação uma única resposta foi considerada sendo que quando houve erro na produção de um ou mais fonemas em uma pseudopalavra resultou em uma pontuação zero.

Os métodos estatísticos utilizados para as análises dos resultados foram o teste de correlação de Spearman e teste de Análise de Variância (ANOVA), sendo o valor do nível de significância menor que $0,05(\mathrm{p}<0,05)$.

\section{RESULTADOS}

Serão descritos os resultados de avaliação obtidos neste estudo separadamente para cada prova: vocabulário, memória, leitura enquanto decodificação e compreensão bem como as correlações entre as variáveis estudadas.

O desempenho de vocabulário pode ser verificado a partir do ABFW - Teste de Linguagem infantil( ${ }^{(17)}$ nos seguintes campos conceituais: vestuário, animais, alimentos, meios de transporte, móveis e utensílios, profissões, locais, formas e cores, brinquedos e instrumentos musicais. Para a análise dos resultados, foram somados os acertos de cada item e transformados em porcentagem; Na avaliação do vocabulário verificou-se que $60 \%$ dos sujeitos ficou acima da média obtida pelo grupo. 
Os sujeitos foram classificados em leitores bons, regulares e ruins de acordo com a Definição Operacional. Apenas duas crianças foram classificadas como leitores regulares pela professora.

Quanto ao desempenho em escrita, para a produção de palavras, $20 \%$ dos sujeitos $(\mathrm{N}=3)$ ficaram abaixo da média para o grupo. Em relação à escrita de pseudopalavras e de frases a partir de figuras, $46 \%(\mathrm{~N}=7)$ ficaram abaixo da média.

$\mathrm{O}$ desempenho acadêmico avaliado pelo TDE indicou que em relação à escrita $73 \%(\mathrm{~N}=11)$ dos alunos foram classificados como pertencentes à série em que se encontram atualmente e/ou acima da mesma. Já em relação à leitura somente $46 \%$ $(\mathrm{N}=7)$ dos alunos apresentaram um nível de leitura de $4^{\mathrm{a}}$ série (Figura 1).

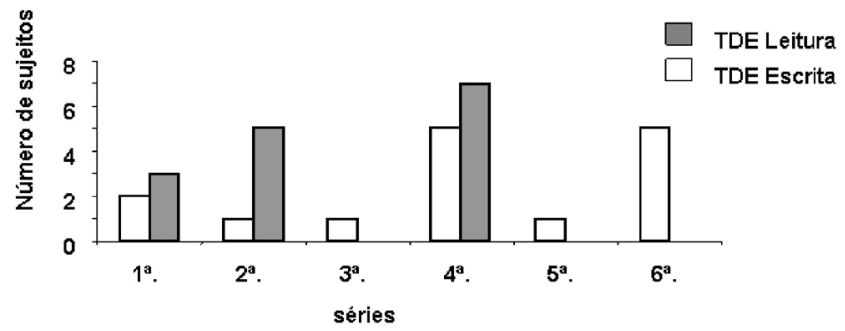

Figura 1. Classificação de sujeitos por nível de escolaridade de acordo com o Teste de Desempenho Escolar - TDE (Escrita e Leitura)

A Figura 2 ilustra a média da taxa de leitura em relação ao nível de leitura do TDE. Quanto à compreensão do texto, apenas $33 \%$ dos alunos, acertaram todas as respostas para o texto 1 e $40 \%$ dos sujeitos para o texto 2 .

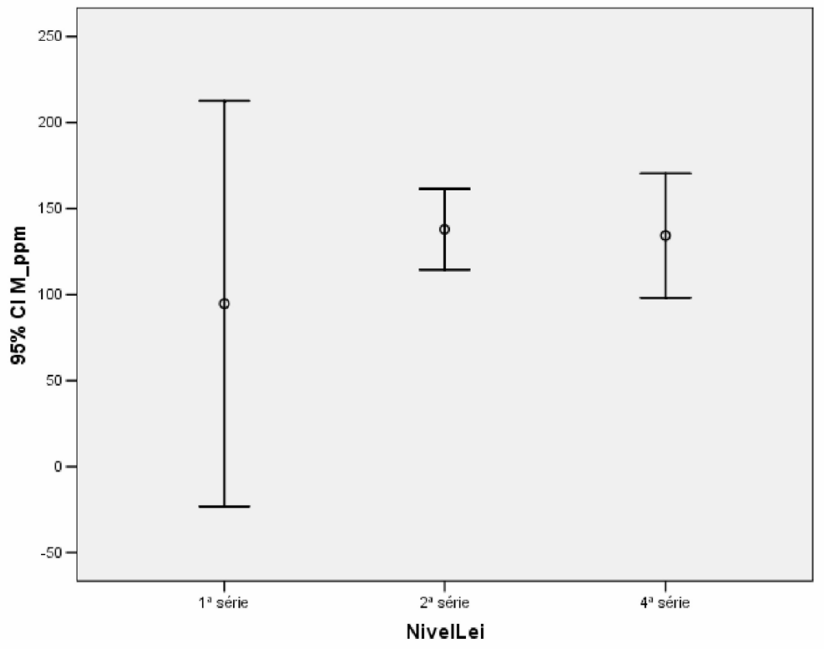

Figura 2. Média da taxa de leitura em palavras por minuto (ppm) em relação à classificação do nível de leitura pelo TDE

Na Figura 3 ilustra-se o desempenho da compreensão avaliado pelo texto cloze de acordo com a classificação do nível de leitura pelo TDE.

A Tabela 1 expõe os resultados dos sujeitos no Teste de Repetição de Pseudopalavras. Apenas um sujeito teve um desempenho abaixo do esperado para a idade de acordo com o padrão de normalidade referido $(\mathrm{N}=36,6)$. Não houve

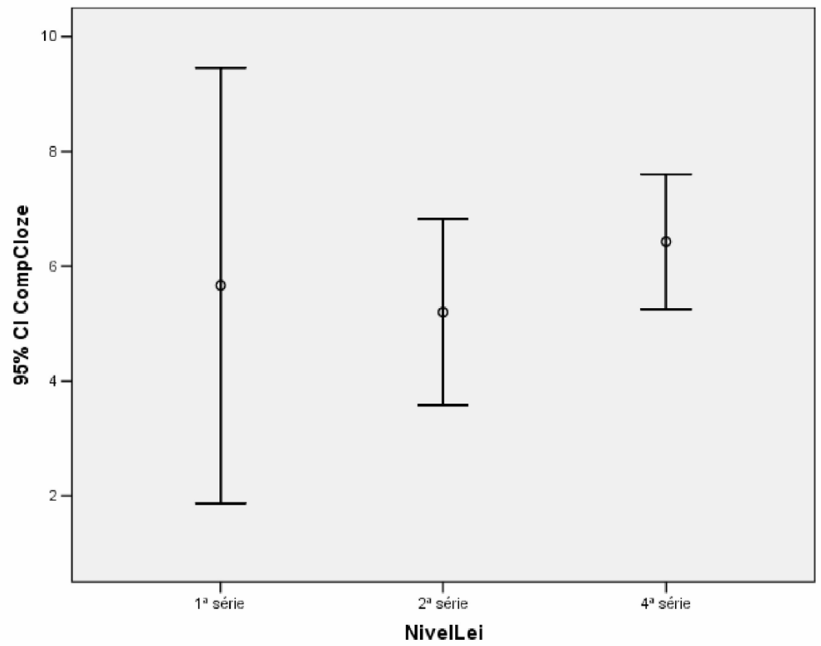

Figura 3. Desempenho de compreensão em relação a classificação do nível de leitura pelo TDE

Tabela 1. Desempenho dos sujeitos no Teste de Repetição de Pseudopalavras

\begin{tabular}{lcc}
\hline Sujeitos & Acertos & Esperado \\
\hline Média & 38,8 & 36,4 \\
DP & 1,2 & 1,4 \\
\hline
\end{tabular}

correlação significante entre a Repetição de Pseudopalavras e compreensão.

A Tabela 2 mostra os resultados dos sujeitos em relação às listas de palavras e pseudopalavras do teste de memória seqüencial auditiva. Em relação ao intervalo de $500 \mathrm{~ms}$ entre as palavras, a média do nível obtido pelos sujeitos foi 3, e no intervalo de 1s, foi 3,1. Já para as listas de pseudopalavras, a diferença entre elas foi maior. A média obtida com o intervalo de $500 \mathrm{~ms}$ entre as pseudopalavras foi de 1,8 , e a média para o intervalo de 1 s foi de 2,3 .

Tabela 2. Desempenho dos sujeitos no teste de memória seqüencial auditiva em relação às listas de palavras e pseudopalavras

\begin{tabular}{lcc}
\hline Estimulo & Intervalo & Média (DP) \\
\hline Palavras & $500 \mathrm{~ms}$ & $3(1,3)$ \\
Palavras & $1000 \mathrm{~ms}$ & $3,1(0,6)$ \\
Pseudopalavras & $500 \mathrm{~ms}$ & $1,8(0,6)$ \\
Pseudopalavras & $1000 \mathrm{~ms}$ & $2,3(0,7)$ \\
\hline
\end{tabular}

A medida de armazenamento de pseudopalavras mostrou uma correlação estatisticamente significante com o tempo de realização do texto cloze (correlação de Spearman, $\mathrm{p}=0,004$ e $r=-0,689$ ) e com o vocabulário (correlação de Spearman, $\mathrm{p}=0,035$ e $\mathrm{r}=0,546)$.

O nível de span calculado foi maior para palavras do que para pseudopalavras (ANOVA, $\mathrm{F}=8,207, \mathrm{p}<0,008$ ), porém não houve um efeito significante do intervalo de apresentação dos estímulos para recordação. A análise do número total de itens recordados no teste evidenciou um efeito de lexicalida- 
de (ANOVA, $\mathrm{F}=8239,53, \mathrm{p}<0,007)$ mas não de intervalo de apresentação.

No teste de memória seqüencial não-verbal, $66 \%(\mathrm{~N}=10)$ dos sujeitos tiveram um desempenho abaixo da média obtida pelo grupo, como mostra a Tabela 3.

Tabela 3. Desempenho dos sujeitos no teste de memória seqüencial não-verbal

\begin{tabular}{lc}
\hline Sujeitos & Nível \\
\hline Média & 7,2 \\
D.P. & 1,6 \\
\hline
\end{tabular}

Quanto maior o tempo para realização do texto cloze melhor foi o desempenho em compreensão (correlação de Spearman, $\mathrm{p}=0,010$ e r= 0,638); quanto melhor o desempenho em vocabulário expressivo melhor a compreensão (correlação de Spearman, $\mathrm{p}=0,001$ e r= $-0,745$ ).

\section{DISCUSSÃO}

O estudo teve como objetivo geral investigar o papel da memória verbal e não-verbal na compreensão de leitura e como objetivos específicos avaliar as habilidades de memória verbal e não-verbal em um grupo de crianças de $4^{\mathrm{a}}$ série e estabelecer a relação entre a memória operacional verbal e não-verbal, vocabulário e a compreensão de leitura.

Houve correlação significante entre a compreensão de leitura e desempenho em vocabulário expressivo, e a memória seqüencial de pseudopalavras, mas não para a memória seqüencial de palavras, contrariando outros estudos em que a compreensão de leitura correlacionou-se com o span de palavras $^{(20)}$. Em outra pesquisa ${ }^{(12)}$ observou-se que o vocabulário e a memória operacional verbal são fortes preditores para a compreensão de leitura. Já em outro estudo ${ }^{(21)}$ não foram encontradas correlações fortes entre memória, habilidades de compreensão e vocabulário. No estudo em questão, a não correlação entre as variáveis pode ter ocorrido pelo fato de se avaliar um novo vocábulo a partir de um contexto, diferentemente do presente trabalho, em que o nível do vocabulário foi verificado a partir de figuras isoladas e apresentadas fora de contexto.

O desempenho de leitura de palavras isoladas, avaliadas pelo TDE, não foi um fator relevante para a compreensão de textos escritos neste grupo de crianças de $4^{\mathrm{a}}$ série, sem queixas de dificuldades escolares. No geral, o desempenho dos sujeitos nesse teste foi bom e, além disso, a Língua Portuguesa possui uma estrutura de fácil decodificação. Alguns autores ${ }^{(22)}$ sugerem que os dois componentes de leitura, compreensão e decodificação, sejam instruídos independentemente devido às diferenças da trajetória do desenvolvimento de ambos. Observando a Figura 3, nota-se que apesar da diferença da média da taxa de leitura não ser significante a variabilidade de desempenho para os sujeitos classificados com nível de leitura para $1^{a}$ série é muito maior; esses resultados podem indicar um processo de transição da rota de leitura.

No presente estudo, a classificação dos alunos quanto ao desempenho de leitura realizada pela professora de $4^{\mathrm{a}}$ série de uma escola particular coincidiu com os resultados obtidos em termos da taxa de leitura dos dois textos. Na Austrália, os professores do pré-primário e da primeira série foram capazes de identificar crianças de risco para dificuldades de linguagem $^{(23)}$. No Brasil, uma pesquisa mostra a discrepância entre a avaliação realizada no estudo com a da professora da rede pública $^{(24)}$. Essas diferenças reforçam a importância de que o sistema educacional invista em programas de capacitação de professores para esse fim, possibilitando que as crianças com risco para dificuldades de aprendizagem possam ser encaminhadas precocemente para avaliação e atendimento especializado.

A compreensão de leitura mostrou uma correlação positiva e significante com o vocabulário, o tempo de realização do texto cloze e a memória operacional verbal, resultados também encontrados em vários trabalhos em outros idiomas e com sujeitos de idades diversas ${ }^{(9-12,20-22)}$.

Esses achados, no entanto, discordam de outro estudo ${ }^{(21)}$, em que não houve correlação estatisticamente significante entre compreensão, memória e vocabulário.

Espera-se que o aluno de $1^{\mathrm{a}}$ a $4^{\mathrm{a}}$ série do Ensino Fundamental, leia de forma independente textos cujo conteúdo e forma são familiares, e demonstre ter compreendido um texto lido por ele ou por alguém de maneira global e não fragmentada, por meio de uma conversa, de um debate, de um reconto ou por escrito ${ }^{(25)}$.

A compreensão de leitura também foi avaliada pelo texto cloze, em que um número maior de crianças foi classificado como tendo uma compreensão ruim, em relação aos dois textos lidos. O uso do texto cloze é a técnica que melhor avalia a compreensão de leitura em crianças ${ }^{(26)}$. Para a realização dessa tarefa, além da memória operacional e do vocabulário, está envolvida a habilidade de realizar inferências ${ }^{(4)}$. O vocabulário, habilidades verbais, memória operacional, habilidades de integração e inferência, monitoração da compreensão e conhecimento estrutural da história devem trabalhar em conjunto para a garantia de uma boa compreensão ${ }^{(13)}$.

O nível de span calculado foi maior para palavras do que para pseudopalavras, porém não houve um efeito significante do intervalo de apresentação dos estímulos para recordação. A análise do número total de itens recordados no teste evidenciou um efeito de lexicalidade, concordando com outro estudo $^{(18)}$, mas não de intervalo de apresentação. O efeito de lexicalidade aponta para o uso de estratégias semânticas para manutenção e recordação dos estímulos. O efeito do intervalo de apresentação não foi observado em crianças desta idade, o que aponta para a necessidade de investigar o desenvolvimento da memória operacional, tanto em termos da complexidade do processamento lingüístico como de recursos cognitivos.

Em outro estudo ${ }^{(27)}$, foi encontrado que o desempenho nas tarefas de memória operacional verbal e visuoespacial possui um crescimento contínuo com pico em aproximadamente 45 anos, e que crianças e adultos mais velhos apresentam nível mais baixo no desempenho da memória operacional relacionada a preservação da nova informação comparado com adultos jovens.

É importante ressaltar que não houve uma correlação estatisticamente significante entre o span de palavras e o span de 
pseudopalavras, porém, com uma amostra maior, esses dados teriam maior relevância. Isso pode ter ocorrido pela facilidade do teste em relação à idade dos sujeitos sem dificuldades.

O span verbal não correlacionou com o span não-verbal. Um possível motivo seria o fato de um estímulo ser lingüístico e o outro auditivo. $\mathrm{O}$ desempenho nas tarefas do teste que avalia o processamento auditivo em crianças com dificuldades escolares e sem queixa melhora com o aumento da idade para ambos os grupos. Embora para o grupo de crianças com dificuldades esse aumento não foi gradual e mais discrepante, portanto crianças com dificuldades escolares podem apresentar atraso na maturação das habilidades auditivas ${ }^{(28)}$. No nosso estudo não foi encontrada uma relação entre habilidades auditivas, do span não-verbal, com a idade das crianças, talvez pelo fato da tarefa ter sido fácil para a população estudada.

A capacidade da alça fonológica é relativamente constante durante o desenvolvimento, embora o aprendizado escolar aumente a eficiência desse sistema ${ }^{(29)}$. Esses autores verificaram um efeito de comprimento de palavras, o que não ocorreu no nosso estudo por se tratar de uma população sem queixa. Nesta pesquisa não foi feito um estudo do tipo de erro cometido pelos sujeitos, pois os mesmos foram muito raros. Sujeitos com boa consciência fonológica têm bom desempenho na exatidão de leitura ${ }^{(9)}$. No nosso estudo o teste de Repetição de Pseudopalavras não correlacionou com nenhum outro teste realizado nesse estudo, o que indica que o mesmo é muito fácil para essa idade, em uma população normal.

O sujeito 12 apresentou um mau desempenho no TDE, em que a classificação do seu nível de leitura e escrita está como $1^{\mathrm{a}}$ série; não teve bom desempenho no span não-verbal e verbal, vocabulário ruim e velocidade de leitura lenta, entretanto teve um bom desempenho na compreensão dos textos 1 e 2, isso indica que as histórias propostas estão fáceis demais para crianças de $4^{\mathrm{a}}$ série sem queixas de compreensão. Neste caso, o tempo de realização do texto cloze (10 minutos) é indicativo de que o sujeito tem dificuldades de compreensão. Portanto a tarefa que melhor avalia a compreensão de leitura é o texto cloze, concordando com outra pesquisa ${ }^{(25)}$.

Os sujeitos 2 e 12 leram ambos os textos com uma taxa lenta, concordando com o julgamento da professora, que os classificou com leitores regulares. Uma avaliação satisfatória da leitura deve constar o nível de competência, o nível cognitivo e biológico (índices das funções cerebrais e sensoriais) $)^{(24)}$.

As dificuldades dos maus leitores, em comparação aos bons leitores, estão relacionadas principalmente ao processamento fonológico, que inclui a consciência fonológica, o vocabulário receptivo auditivo, e a memória fonológica ${ }^{(30)}$. É necessário investigar a função da memória operacional em relação às habilidades de compreensão de textos e com o desempenho em habilidades escolares em uma amostra maior e em vários níveis de escolaridade. Assim, será possível o desenvolvimento de programas de promoção da fluência de leitura apropriados para cada série e para a realidade brasileira.

\section{CONCLUSÕES}

O desempenho em memória operacional não-verbal bem como o desempenho em decodificação de leitura não influenciou no desempenho de compreensão de leitura no grupo estudado. Quanto melhor o nível de vocabulário expressivo mais competente foi a compreensão de leitura nesses sujeitos. A capacidade da memória operacional verbal contribuiu para a compreensão de leitura neste grupo de crianças de $4^{\mathrm{a}}$ série do Ensino Fundamental.

\begin{abstract}
Purpose: To investigate the role of verbal and non-verbal working memory and vocabulary levels in reading comprehension. The specific goals were to evaluate working memory abilities, vocabulary and reading skills in a group of fourth grade children and to estimate the relations between memory comprehension performances. Methods: Participants were 15 children between 9:11 and 11:4 years old with no auditory, visual and/or neurological deficits. All subjects were tested individually for academic performance, reading (decoding and comprehension), vocabulary, and verbal and non-verbal memory. Results: Vocabulary knowledge ( $\mathrm{r}=0,546, \mathrm{p}=0,035)$ and verbal working memory $(r=-0,689, p=0,004)$ showed statistically significant correlations with performance in comprehension in this group of fourth grade children, with no school difficulties. Neither non-verbal working memory nor decoding skills influenced reading comprehension. Conclusion: The performance on vocabulary and the good ability of verbal working memory were relevant variables for reading comprehension in this population.
\end{abstract}

Keywords: Reading; Memory; Comprehension; Students; Education, primary and secondary; Child

\title{
REFERÊNCIAS
}

1. Capovilla FC, organizador. Os novos caminhos da alfabetização infantil: relatório encomendado pela Câmara dos Deputados ao Painel Internacional de Especialistas em Alfabetização Infantil. 2a ed. rev. São Paulo: Memnon; 2005.
2. Navas ALGP, Santos MTMS. Terapia da linguagem escrita. In: Santos MTM, Navas ALGP, Santos MTMS, organizadores. Distúrbios de leitura e escrita: teoria e prática. Barueri: Manole; 2004. p.191-223. 
3. McNamara DS, Floyd RG, Best R, Louwerse M. World knowledge driving young readers' comprehension difficulties. International Conference on Learning Sciences. Proceedings of the 6th international conference on Learning sciences. Santa Monica, California; 2004. p.326-33.

4. Cain K, Oakhill JV, Barnes MA, Bryant PE. Comprehension skill, inference-making ability, and their relation to knowledge. Mem Cognit. 2001;29(6):850-9.

5. Caccamise D, Synder L. Theory and pedagogical practices of text comprehension. Top Lang Disord. 2005;25(1):5-20.

6. National Institute of Child Health and Human Development. (2000). Report of the National Reading Panel. Teaching children to read: An evidence-based assessment of the scientific research literature on reading and its implications for reading instruction. Washington, DC: U.S. Government Printing Office. [NIH Publication No. 00-4769].

7. Baddeley AD. Working memory. Oxford: Oxford University Press; 1986.

8. Gathercole SE. Cognitive approaches to the development of short-term memory. Trends Cogn Sci.1999;3(11):410-9.

9. Leather CV, Henry LA. Working memory span and phonological awareness tasks as predictors of early reading ability. J Exp Child Psychol. 1994;58(1):88-111.

10. Swanson HL, Berninger V. The role of working memory in skilled and less skilled readers' comprehension. Intelligence. 1995;21(1):83-108.

11. Palladino P, Cornoldi C, De Beni R, Pazzaglia F. Working memory and updating processes in reading comprehension. Mem Cognit. 2001;29(2):344-54.

12. Seigneuric A, Ehrlich MF, Oakhill JV, Yuill NM. Working memory resources and children's reading comprehension. Read Writ. 2003;13(1-2):81-103.

13. Cain K, Bryant P, Oakhill J. Children's reading comprehension ability: concurrent prediction by working memory, verbal ability, and component skills. J Educ Psychol. 2004;96(1):31-42.

14. Gathercole SE, Alloway TP, Wilis C, Adams AM. Working memory in children with reading disabilities. J Exp Child Psychol. 2006;93(3):265-81.

15. Pinheiro AMV, Costa AEB. Escala de avaliação de competência de leitura pelo professor. In: VII Encontro Mineiro de Avaliação Psicológica - EMAP, 2005, Belo Horizonte. Livro de resumos do VII Encontro Mineiro de Avaliação Psicológica - EMAP, 2005. p. 27

16. Stein LM. Teste de desempenho escolar: manual para aplicação e interpretação. São Paulo: Casa do Psicólogo; 1994.
17. Befi-Lopes DM. Vocabulário. In: Andrade CRF, Befi-Lopes DB, Fernandes FDM, Wertzner HF. ABFW - teste de linguagem infantil nas áreas de fonologia, vocabulário, fluência e pragmática. 2a ed rev. ampl. e atual. Barueri: Pró-Fono; 2004. cap. 2.[ Anexo 4].

18. Navas ALPG, Ferraz EC, Giangiacomo MCPB, Satake TKR. Efeito de lexicalidade e de intervalo de apresentação em uma tarefa de span de palavras e pseudopalavras. In: Anais do VIII Congresso Brasileiro de Fonoaudiologia. Santos, 2005.

19. Santos FH, Bueno OF. Validation of the Brazilian Children's Test of Pseudoword Repetition in Portuguese speakers aged 4 to 10 years. Braz J Med Biol Res. 2003;36(11):1533-47.

20. Engle RW, Cantor J, Carullo JJ. Individual differences in working memory and comprehension: a test of four hypotheses. J Exp Psychol Learn Mem Cogn. 1992;18(5):972-92.

21. Cain K, Lemmon K, Oakhill J. Individual differences in the inference of word meanings from context: the influence of reading comprehension, vocabulary knowledge, and memory capacity. J Educ Psychol. 2004;96(4):671-81.

22. Oakhill JV, Cain K, Bryant PE. The dissociation of word reading and text comprehension: evidence from component skills. Lang Cognit Process. 2003;18(4):443-68.

23. Willians C. Teacher judgements of the language skills of children in the early years of schooling. Child Lang Teach Ther. 2006;22(2):135-54.

24. Pinheiro AMV. Heterogeneidade entre leitores julgados competentes pelas professoras. Psicol Reflex Crit. 2001; 14(3): 537-51.

25. Brasil. Ministério da Educação. Parâmetros Curriculares Nacionais [Internet]. Brasília(DF):Ministério da Educação; c2003. [citado 2007 Set 10]. Disponível em http://portal.mec.gov.br/seb/arquivos/pdf/livro02. pdf

26. Joly MCRA, Nicolau AF. Avaliação de compreensão em leitura usando

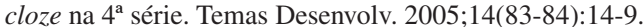

27. Swanson HL. What develops in working memory? A life span perspective. Dev Psychol. 1999;35(4):986-1000.

28. Neves IF, Schochat E. Maturação do processamento auditivo em crianças com e sem dificuldades escolares. Pró-Fono. 2005;17(3):311-20.

29. Santos FH, Bueno OF, Gathercole SE. Errors in nonword repetition: bridging short- and long- term memory. Braz J Med Biol Res. 2006;39(3):371-85.

30. Capovilla AGS, Capovilla FC, Suiter I. Processamento cognitivo em crianças com e sem dificuldades de leitura. Psicol Estud. 2004;9(3):449-58. 